\title{
Análisis del control de la comprensión lectora en textos científicos en alumnos de Secundaria Obligatoria y Bachillerato ${ }^{1}$
}

\section{Analysis of the reading and comprehension control in scientific texts in high school students}

\author{
Lourdes JIMÉNEZ TARACIDO, Daniela BARIDON CHAUVIE y \\ Ana Isabel MANZANAL MARTÍNEZ \\ Universidad Internacional de La Rioja
}

Recibido: Julio 2014

Evaluado: Noviembre 2014

Aceptado: Noviembre 2014

\section{Resumen}

El proceso de aprendizaje de un alumno puede verse afectado negativamente cuando su control de la comprensión lectora no es adecuado. Esta investigación se centra en cómo una muestra de alumnos de Educación Secundaria Obligatoria (ESO) y Bachillerato evalúan su comprensión lectora con textos científicos manipulados. El instrumento fue diseñado ad hoc para establecer qué nivel de control de la comprensión alcanzan los alumnos cuando leen textos cortos del área de ciencias con una contradicción añadida. Los resultados indican que en los cursos de $1^{\circ}$ y $3^{\circ}$ de ESO, predomina una evaluación y/o regulación inadecuada, en cambio en $1^{\circ}$ de Bachillerato la evaluación y regulación adecuada es más frecuente. Por otra parte, no se han encontrado diferencias significativas por el tipo de texto, en relación al curso o género. Finalmente, las correlaciones entre el control de la comprensión y las calificaciones escolares indican una asociación débil e inversamente proporcional a la edad de los educandos, resultados que concuerdan con los estudios previos.

Palabras clave: metacognición, educación secundaria obligatoria y bachillerato, metacomprensión, control de la comprensión.

\begin{abstract}
Students' learning process can be negatively affected when their reading and comprehension control is not appropriated. This research focuses on the analysis of how a group of students from high school evaluate their reading comprehension in manipulated scientific texts. An analysis tool was designed to determine the students' degree of comprehension control when

\footnotetext{
${ }^{1}$ Esta actividad ha sido parcialmente financiada por UNIR Research (http://research.unir.net), Universidad Internacional de la Rioja (UNIR, http://www.unir.net), dentro del Plan Propio de Investigación, Desarrollo e Innovación [2013- 2015], Grupo de Investigación Educación Personalizada en la Era Digital (EPEDIG).
} 
reading a scientific short text with an added contradiction. The results have revealed that the students from 1st and 3rd ESO do not properly self-evaluated their reading comprehension. A different behavior has been observed in 1st Bachillerato, where appropriate evaluation and regulation seem to be more frequent. Moreover, no significant differences have been found regarding the type of text, year or gender. Finally, as identified by previous research, the correlations between the students' comprehension control and their school marks have shown to have a weak relationship and inversely proportional to the students' age.

Keywords: metacognition, high school, metacomprehension, control of comprehension.

Esta investigación evalúa el control de la comprensión lectora en una muestra de alumnos de ESO y Bachillerato utilizando el paradigma de detección del error en textos cortos científicos.

La finalidad es identificar las destrezas metacognitivas que utilizan los alumnos ante la tarea de leer un texto e identificar la relación con el rendimiento académico medido según las calificaciones escolares. El estudio de la metacognición tiene dos aspectos clave. Uno de ellos es la capacidad de detectar si existe algún problema de comprensión y, el segundo, y alcanzado el primero, es si el sujeto es capaz de establecer los mecanismos para superar estas dificultades comprensoras. Por tanto, las estrategias metacognitivas son aquellas que pueden ayudar a detectar dificultades de comprensión, siendo el control de la comprensión una de ellas.

La importancia de que los alumnos posean y apliquen estas estrategias metacognitivas radica en la influencia que tienen para lograr una comprensión adecuada sobre lo que leen. Si el alumno sabe que no ha comprendido aquello que ha leído podrá poner en marcha estrategias de reparación (búsqueda de palabras desconocidas, formulación de preguntas, etc.). En cambio, si el alumno "no sabe que no sabe" (Campanario y Moya, 1999, p. 180) no podrá actuar en consecuencia y la comprensión se verá seriamente comprometida.

En estudios empíricos, con estudiantes entre 12 y 18 años, donde se han usado textos científicos con inconsistencias, se ha demostrado que muchos alumnos no son capaces de detectarlas y, en algunos casos, tratan de justificarlas con razonamientos nada científicos (Otero y Campanario, 1990; Otero, Campanario y Hopkins, 1992). Asimismo, estos estudios han revelado una débil relación entre el control de la comprensión y el rendimiento académico. Según estos autores, esto puede ser debido al escaso valor que se le otorga en la evaluación al aprendizaje significativo frente al memorístico.

Considerando que la mayor parte de los conocimientos y aprendizajes de índole científico se trasmiten a través de los textos escritos (Muñoz-Calvo, Muñoz-Muñoz, García y Granado, 2013) se justifica el interés generado en la Didáctica de las Ciencias (Georghiades, 2004; García-Arista, Otero, Caldeira, Prata-Pina, Patricio y Cardoso, 2006) por la investigación de esta y otras estrategias metacognitivas. 


\section{La comprensión lectora}

El control de la comprensión lectora permite al lector tener un conocimiento declarativo y procedimental de las acciones que lleva a cabo mientras lee (Mazzitelli, Maturano y Macías, 2007). Su importancia reside en la influencia que ejerce en el éxito o fracaso a la hora de abordar una tarea donde interviene una actividad relacionada con la lectura.

La relación entre el control de la comprensión y las competencias clave, definidas por la Organización para la Cooperación y el Desarrollo Económico (OCDE), es manifiesta ya que un buen control de comprensión mejora la comprensión lectora y, esta a su vez, se ha definido como una competencia genérica, pues hace posible "un buen rendimiento académico, el aprendizaje autónomo y la transferencia del conocimiento" (Guevara y Guerra, 2013, p. 324). Por tanto, un buen control de la comprensión se posiciona como una estrategia con un valor relevante en el aprendizaje permanente, tal y como establecen las directrices del marco europeo de educación. En este sentido, Washer (2007) y López (2007) tras sus investigaciones con alumnos universitarios atribuyen a la metacognición un valor importante para el educando ya que le puede ayudar a aprender a aprender y a la adquisición de competencias genéricas.

La naturaleza de la comprensión lectora ha sido estudiada desde diferentes ámbitos con el propósito de hallar estrategias que conduzcan a la adquisición de la competencia lectora. Las características del alumno, del texto, de la tarea y del contexto ejercen influencia en la comprensión del texto (Alexander y Kulikowich, 1994; Sanjosé, Fernández y Vidal-Abarca, 2010).

Las investigaciones de Guevara y Guerra (2013) centradas en el aprendiz afirman que una mejor o peor comprensión lectora está relacionada con aspectos motivacionales tales como autoconcepto, autoeficacia y tipo de metas del lector. En este sentido, sujetos con una motivación intrínseca hacia el aprendizaje y metas centradas en la tarea presenta mejores resultados ante pruebas que requieren ejercer esta capacidad, ya que estos educandos ponen en marcha estrategias profundas de aprendizaje que les lleva a leer más, mejorando a su vez su comprensión lectora.

\section{Control de la comprensión}

Las estrategias de comprensión lectora pueden dividirse según el momento en el que se activen Así se distingue entre estrategias previas a la lectura (formular hipótesis), durante la misma (control de la comprensión) y tras esta (subrayado) (Cooper, 1990).

El auto-monitoreo de la comprensión se incluye en la categoría de estrategias de procesamiento profundo (Cano, 2005). Guevara y Guerra (2013) califican esta estrategia metacognitiva como una de las mejores técnicas que se pueden utilizar para favorecer el control de la comprensión, ya que así, el sujeto puede detectar si ha aprendido o, si por el contrario ha detectado errores y carencias. 
Según Winograd y Johnston (1982), el conocimiento que aporta esta monitorización de la comprensión es importante "porque proporciona información vital para el sujeto acerca de la eficacia de su comportamiento lector" (p. 62). Así, el lector que controla su propia comprensión tiene una base óptima para elegir la mejor estrategia lectora que necesita en cada momento. Por tanto, para mejorar la comprensión lectora será necesario fomentar estrategias que incidan en las destrezas de control y regulación de la comprensión, términos que hacen alusión a la metacognición descrita por Flavell en 1976. La metacognición se define como el "conocimiento que uno tiene acerca de los propios procesos y productos cognitivos o cualquier otro asunto relacionado con ellos" (Flavell, 1976, p. 232).

Para evaluar el control de la comprensión existen diferentes estrategias de monitorización, siendo el paradigma de detección del error (Baker, 1979 citado por Winograd y Johnston, 1982) una de ellas. En este modelo, se pide a los sujetos que realicen una tarea donde se ofrece un texto manipulado que incluye un error. Si el sujeto no menciona el error después de la lectura, se solicita que explique la causa en un intento de saber por qué no lo ha mencionado. Se pueden utilizar diferentes tipos de errores tales como inclusión de pasajes desorganizados, falta de información, frases fuera de contexto o la incorporación de frases contradictorias (Baker, 1979, citado por Winograd y Johnston, 1982).

Otero y Campanario (1990) y Otero et al. (1992), en base al modelo de Kintsch y van Dijk de 1983, utilizaron el paradigma de la contradicción para analizar la influencia de las variables metacognitivas en el aprendizaje de los estudiantes a partir de la lectura de textos científicos y su relación con el rendimiento académico. Los resultados obtenidos remarcan la relevancia de las estrategias metacognitivas en el aprendizaje de las ciencias puesto que, tal como afirman los autores, "las dificultades que tienen los estudiantes para controlar su propia comprensión pueden ser tan importante como las dificultades para una comprensión correcta" (Otero y Campanario, 1990, p.457) en referencia al peso de lo metacognitivo sobre lo cognitivo. Con respecto al rendimiento académico (computado como calificaciones escolares), estos autores detectaron que la capacidad para monitorear la comprensión (en sus siglas CMA de Comprehension Monitoring Ability) estaba relacionada significativamente con dicha variable. Aunque las correlaciones, entre rendimiento académico y control de la comprensión reveladas eran débiles, decrecían con la edad de los estudiantes y no presentaban variaciones según el género, coincidiendo con los trabajos de Zimmerman y Martínez-Pons (1988).

\section{Metacomprensión lectora}

El monitoreo y la regulación de la lectura, también llamados metacomprensión, son aspectos clave porque se refieren a los mecanismos de control que se usan durante la comprensión lectora (Mokhtari y Reichard, 2002), permitiendo al lector ser autónomo y tener conciencia sobre la tarea que está llevando a cabo, identificado las dificultades y la forma de mejorar la resolución de la misma (Mazzitelli et al., 2007). 
El control de la comprensión mide la capacidad del alumno para detectar contradicciones e incoherencias lógicas, evaluar el propio rendimiento y determinar lo que no se sabe. La comprensión implica dos fases interrelacionadas. Tras la lectura, decidir si se ha entendido o no (evaluación de la comprensión) y, si hubiera detectado problemas de comprensión, tomar medidas para corregirla mediante estrategias reparadoras, como releer el texto o buscar información externa (Otero, 1992 y 1998).

\section{Fases de la metacomprensión: evaluación y regulación}

Durante la fase de evaluación, el lector juzga si ha comprendido o no el texto y para ello, es esencial que sea capaz de detectar las dificultades o errores en lo que lee (Otero y Graesser, 2001). Los juicios de metacomprensión del lector para ser correctos deben estar basados en el nivel real de comprensión, y así evitar situaciones donde por ejemplo, el estudiante revise innecesariamente un texto bien comprendido o, lo que es peor, estudie un texto malentendido.

Durante la fase de regulación, el lector puede poner en marcha acciones encaminadas a reparar los errores detectados en el texto, como son la relectura para aclarar puntos confusos o la búsqueda de significados de palabras desconocidas (Baker, 1985). El conocimiento de estas estrategias reparadoras no es sinónimo de su utilización. Si el lector no las utiliza, persiste la falta de coherencia en la representación mental del texto y finalmente, se compromete el aprendizaje. Solo es posible una representación coherente una vez que las inconsistencias sean detectadas (evaluación) y corregidas (regulación) (Otero, 1998).

Como afirman Mazzitelli et al., (2007) una regulación inadecuada "es un problema, no solo en la comprensión de textos sino que compromete todos los aprendizajes" (p. 218).

\section{Control de la comprensión y rendimiento académico}

A la vista de lo expuesto anteriormente, se justifica el interés por investigar la correspondencia entre comprensión, autorregulación y rendimiento académico. Un estudio de Pintrich y De Groot (1990) concluye que los alumnos con un buen control de la comprensión tienen mayor éxito académico. Posteriores investigaciones en este sentido, indican que las estrategias cognitivas y metacognitivas son buenos predictores del rendimiento escolar (Broc, 2012; Rodríguez, 2009; Solaz y Sanjosé, 2008; Zimmerman y Martínez- Pons, 1988).

Un alumno que confía en sus capacidades y su autoeficacia (autocompetente) se compromete de forma activa en su proceso de aprendizaje, lo que determina las estrategias cognitivas y metacognitivas que pone en funcionamiento cuando aborda las tareas que, a su vez inciden de forma directa y positiva sobre el rendimiento académico. Así pues, el sujeto es consciente de la utilidad del proceso de autorregulación de cara a aumentar el éxito académico (Schunk y Zimmerman, 1994). Por ejemplo, Solaz y Sanjosé (2008) señalan el trabajo de Teong de 2003 en que se 
evidencia que los alumnos entrenados en estrategias metacognitivas resuelven los problemas que se les proponen a nivel educativo de forma más eficiente que aquellos alumnos que no están entrenados.

Cuando se estudia el rendimiento académico dentro el área de las ciencias (Otero et al., 1992; García-Arista et al., 2007) la relación de este con el control de la comprensión se debilita, e incluso los resultados parecen indicar que las destrezas metacognitivas tienen un papel limitado ya que otras variables lo condicionan.

\section{Objetivos}

En la presente investigación el objetivo general planteado es evaluar el control de la comprensión (evaluación y regulación) de textos científicos manipulados en una muestra de alumnos de ESO y Bachillerato, y como objetivos específicos se señalan:

1. caracterizar la muestra de estudio según el binomio evaluación-regulación.

2. identificar si las variables curso y género, influyen en el grado de comprensión.

3. relacionar el control de la comprensión total con el rendimiento académico.

\section{Método}

\section{Participantes}

El centro educativo participante en la investigación es de titularidad privada, confesional y concertado en todos sus niveles, desde $2^{\circ}$ Ciclo de Infantil hasta $2^{\circ}$ de Bachillerato. Su población estudiantil presenta amplia diversidad en cuanto a las nacionalidades de procedencia de chicos y chicas.

La muestra está formada por 134 estudiantes pertenecientes a grupos de $1^{\circ}$ de ESO A y B, de $3^{\circ}$ de ESO A y B, y de $1^{\circ}$ de Bachillerato. La distribución de alumnos por curso se presenta en la tabla I.

La distribución del total de estudiantes participantes por género, corresponde a un $48,5 \%$ de mujeres, y un $51,5 \%$ de hombres.

\begin{tabular}{cccc}
\hline Grupo & Mujeres & Hombres & Total \\
\hline \multirow{2}{*}{$1^{\circ}$ ESO } & 20 & 21 & 41 \\
& $(48,8 \%)$ & $(51,2 \%)$ & $(30,6 \%)$ \\
\hline \multirow{2}{*}{$3^{\circ} \mathrm{ESO}$} & 33 & 25 & 58 \\
& $(56,9 \%)$ & $(43,1 \%)$ & $(43,3 \%)$ \\
\hline \multirow{2}{*}{$\mathbf{1}^{\circ} \mathrm{BACH}$} & 12 & 23 & 35 \\
& $(34,3 \%)$ & $(65,7 \%)$ & $(26,1 \%)$ \\
\hline \multirow{2}{*}{ Total } & 65 & 69 & 134 \\
& $(45,5 \%)$ & $(51,5 \%)$ & $(100 \%)$ \\
\hline
\end{tabular}

Tabla I. Distribución de alumnos por grupo de pertenencia y género (\% respecto al total). 


\section{Materiales y Medidas}

El instrumento construido ad hoc permite medir el control de la comprensión de los estudiantes por medio de textos científicos cortos con inconsistencias. Dicho instrumento corresponde a una adaptación del creado por Otero y Campanario (1990), y utilizado en sus investigaciones (Otero et al., 1992; García-Arista et al., 2006).

El instrumento consta de dos partes que no se utilizan de forma simultánea. El cuadernillo 1 contiene cuatro textos científicos cortos de diferentes temas de ciencias de 60 a 101 palabras cada uno. Tres de los cuatro textos contienen dos frases contradictorias (inconsistencias), situadas siempre en la segunda y última frase del párrafo. El cuadernillo 2 presenta los mismos textos con las contradicciones, en este caso, destacadas en color rojo. Los temas elegidos para estos textos son: Algas, Medusas, Diversidad Bacteriana y Fouling.

La tabla II propone a modo de ejemplo uno de los textos con contradicción, correspondiente al cuadernillo 1 , para los grupos de $1^{\circ}$ de ESO:

Las medus as

Las medusas son invertebrados translúcidos que en oportunidades en verano, invaden las costas. Las medusas podrían beneficiarse con el cambio climático. Esto se debe, a que en verano aumenta la temperatura y la salinidad de las costas, y esto permite que las medusas avancen en las aguas costeras. Con lo cual, las medusas podrían ser perjudicadas con el cambio climático.

Tabla II. Texto científico corto con contradicción para $1^{\circ}$ de ESO.

La temática de los cuatro textos del cuadernillo 1 se mantiene en los tres niveles, si bien, basándonos en trabajos previos (Otero y Campanario, 1990; Otero et al., 1992) la complejidad del texto aumenta para $3^{\circ}$ de ESO y $1^{\circ}$ de Bachillerato

. El texto 2 del cuadernillo 1 es sin contradicción (Tabla III), con la finalidad de medir la comprensibilidad de los textos y garantizar que los resultados medidos en los textos manipulados fueran fruto de las habilidades de control de la comprensión de los estudiantes y no de una dificultad cognitiva de los textos.

Las medusas

Las medusas son invertebrados translúcidos que en oportunidades en verano, invaden las costas. Las medusas podrían beneficiarse con el cambio climático. Esto se debe, a que en verano aumenta la temperatura y la salinidad de las costas, y esto permite que las medusas avancen en las aguas costeras. Su presencia en las playas se ha triplicado en los últimos años.

Tabla III. Texto científico corto sin contradicción para $1^{\circ}$ de ESO.

En el cuadernillo 1, cada texto es acompañado de una escala Likert de 1 a 4 con indicaciones para que los alumnos señalen si el texto "no se entiende (1), se entiende 
con dificultad (2), se entiende relativamente bien (3) o, se entiende muy bien (4)”. Además se solicitó a los alumnos que, en caso de detectar dificultades en el texto, subrayaran la/las frases con dificultad, y explicaran de forma cualitativa la dificultad encontrada.

En relación al cuadernillo 2, este presenta los mismos textos con contradicciones pero evidenciadas en rojo (Tabla IV). Se consulta a los alumnos al respecto por medio de las siguientes preguntas: “a) ¿Al leer te diste cuenta de esta contradicción?”, a responder con Sí o con No. "b) Si has contestado que sí, ¿̇lo indicaste en la prueba anterior?”, a responder también con Sí o con No. Por último, “c) Si te diste cuenta, pero no lo indicaste: ¿por qué no lo hiciste? Por favor, justifica tu respuesta, a continuación”.

Las medusas

Las medusas son invertebrados translúcidos que en oportunidades en verano, invaden las costas. Las medusas podrían beneficiarse con el cambio climático. Esto se debe, a que en verano aumenta la temperatura y la salinidad de las costas, y esto permite que las medusas avancen en las aguas costeras. Con lo cual, las medusas podrían ser perjudicadas con el cambio climático.

Tabla IV. Texto científico corto con contradicción señalada para $1^{\circ}$ de ESO.

La medición de la estrategia metacognitiva de control de la comprensión (en adelante CC) permite conocer si el alumno comprende o no el texto que lee. Aplicar este instrumento con dos cuadernillos (en el cuadernillo 1 el alumno dice si comprende o no y en el cuadernillo 2 se explica) permite contrastar la respuesta inicial del alumno en el cuadernillo 1 con la del cuadernillo 2, y verificar si realmente ha habido comprensión.

Siguiendo la línea de investigación de Otero y Campanario (1990), se evalúa un CC global a partir de la suma del CC de cada uno de los textos. Las categorías para la evaluación de las respuestas de los alumnos se presentan a continuación en la tabla V. 


\begin{tabular}{|c|c|c|c|}
\hline $\begin{array}{l}\text { EVALUACION } \\
\text { DELA } \\
\text { COMPRENSION }\end{array}$ & $\begin{array}{l}\text { REGULACION } \\
\text { DELA } \\
\text { COMPRENSION }\end{array}$ & $\begin{array}{l}\text { CATEGORIAS } \\
\text { GENERALES }\end{array}$ & $\begin{array}{l}\text { Grado de comprensibilidad declarado (escala } \\
\text { Likert) }\end{array}$ \\
\hline \multirow{2}{*}{$\begin{array}{l}\text { I. Inadecuada, no } \\
\text { se detecta el } \\
\text { error }\end{array}$} & \multirow{2}{*}{ Ausente } & $\begin{array}{l}\text { CI } \\
\text { (Conocimiento } \\
\text { lusorio) }\end{array}$ & $\begin{array}{l}\text { No detectan la dificultad pero manifiestan una buena } \\
\text { comprensión del texto o enunciado (Puntuación 3-4) }\end{array}$ \\
\hline & & $\begin{array}{l}\text { DB } \\
\text { (Dificultades } \\
\text { básicas) }\end{array}$ & $\begin{array}{l}\text { No detectan la dificultad pero manifiestan } \\
\text { insatisfacción con la comprensión del texto o } \\
\text { enunciado (Puntuación 1-2) }\end{array}$ \\
\hline \multirow{2}{*}{ II. Adecuada } & \multirow{2}{*}{ Inadecuada } & $\begin{array}{l}\text { FR } \\
\text { (Falta de } \\
\text { regulacion) }\end{array}$ & $\begin{array}{l}\text { Son conscientes de la dificultad (según manifiestan } \\
\text { en la prueba 2) pero no subrayan ni explican el } \\
\text { problema (contradicciones ofalta de información) }\end{array}$ \\
\hline & & $\begin{array}{l}\text { RI } \\
\text { (Regulación } \\
\text { inadecuada) }\end{array}$ & $\begin{array}{l}\text { Son conscientes de la dificultad (según manifiestan } \\
\text { en la prueba 2) y tratan de explicar la dificultad pero } \\
\text { erróneamente }\end{array}$ \\
\hline \multirow[t]{2}{*}{ III. Adecuada } & \multirow[t]{2}{*}{ Adecuada } & $\begin{array}{l}\text { DI(Dificultad } \\
\text { infravalorada) }\end{array}$ & $\begin{array}{l}\text { Detectan la dificultad y la señalan en el texto/ } \\
\text { enunciado problema, la explican pero consideran } \\
\text { buena la comprensión del texto enunciado } \\
\text { (Puntuación 3-4) }\end{array}$ \\
\hline & & $\begin{array}{l}\text { DR } \\
\text { (Detección y } \\
\text { rechazo) }\end{array}$ & $\begin{array}{l}\text { Detectan la dificultad señalándola en el } \\
\text { texto/enunciado la explican y consideran } \\
\text { imsatisfactoria su comprensión (Puntuación 1-2) }\end{array}$ \\
\hline
\end{tabular}

Tabla V. Categorías de análisis

Fuente: Elaborada a partir de Otero y Campanario (1990).

Una vez categorizadas las respuestas, se asigna una puntuación de CC en relación a la comprensibilidad y categoría de la respuesta, de acuerdo a la tabla VI:

\begin{tabular}{ccc}
\hline Categoría & $\begin{array}{c}\text { Puntuación de } \\
\text { comprensibilidad }\end{array}$ & Puntuación de CC \\
\hline Conocimiento ilusorio (CI) & $3-4$ & $0-0$ \\
\hline Dificultades básicas (DB) & $1-2$ & $1-1$ \\
\hline Falta de regulación (FR) & $4-3$ o menos & $2-3$ \\
\hline Regulación inadecuada (RI) & $4-3$ o menos & $4-5$ \\
\hline Dificultad infravalorada (DI) & $4-3$ & $6-7$ \\
\hline Detección y rechazo (DR) & $2-1$ & $8-9$ \\
\hline
\end{tabular}

Tabla VI. Escala de la capacidad de control de la comprensión.

Fuente: Otero et al. (1992, p. 424). 
Cabe destacar que los textos incluidos en este instrumento, de elaboración propia, se referían a temáticas desconocidas por los alumnos, buscando evitar así que los conocimientos previos se interpusieran en el CC. Dichos conocimientos previos podrían condicionar la evaluación de la comprensión del texto, aportando una medida de "consistencia externa”, en vez de medir la "consistencia interna” (Baker, 1985). Esto significa que los alumnos utilizarían lo que ya saben para decidir si el texto se entiende o no, en vez de verificar la coherencia de las ideas expuestas en el texto, que es lo que aquí se espera conocer. Asimismo y con el fin de evaluar la comprensibilidad de los textos, más allá de presentar o no presentar contradicción y de la posición que tuvieran en el cuadernillo, se crearon 4 versiones de cada cuadernillo: alfa, beta, gamma y delta. En cada versión se varió la posición de los textos con contradicción (A, $\mathrm{B}, \mathrm{C}, \mathrm{y} \mathrm{D})$ y sin contradicción $\left(\mathrm{A}^{\mathrm{sc}}, \mathrm{B}^{\mathrm{sc}}, \mathrm{C}^{\mathrm{sc}}, \mathrm{D}^{\mathrm{sc}}\right)$, como se muestra en la tabla VII:

\begin{tabular}{ccccc}
\hline $\begin{array}{c}\text { Versiones/ } \\
\begin{array}{c}\text { Posiciones en } \\
\text { el texto }\end{array}\end{array}$ & alfa & beta & gamma & delta \\
\hline $\mathbf{1}$ & $\mathrm{B}$ & $\mathrm{C}$ & $\mathrm{D}$ & $\mathrm{A}$ \\
\hline $\mathbf{2}$ & $\mathrm{A}^{\mathrm{sc}}$ & $\mathrm{B}^{\mathrm{sc}}$ & $\mathrm{C}^{\mathrm{sc}}$ & $\mathrm{D}^{\mathrm{sc}}$ \\
\hline 3 & $\mathrm{C}$ & $\mathrm{D}$ & $\mathrm{A}$ & $\mathrm{B}$ \\
\hline 4 & $\mathrm{D}$ & $\mathrm{A}$ & $\mathrm{B}$ & $\mathrm{C}$ \\
\hline
\end{tabular}

Tabla VII. Combinación de textos en diferentes versiones de cuadernillo 1.

En el caso del cuadernillo 2, se mantuvieron tales combinaciones, mostrando cada cuadernillo los tres textos con contradicción señaladas, y omitiendo la presencia del texto sin contradicción.

\section{Procedimiento}

Se realizó una presentación clara y concisa del instrumento. Para su aplicación se destinó un tiempo de 30 minutos en cada grupo. Se remarcó que, en ningún caso, se les estaba poniendo a prueba, siendo la finalidad de la misma evaluar los textos para su participación en libros de textos para futuros estudiantes. Se insistió en la importancia de la atención y sinceridad a la hora de realizar la prueba, así como en el anonimato de las mismas.

A continuación se entregó a cada alumno el cuadernillo 1, repartiendo aleatoriamente los diferentes modelos de este (alfa, beta, gamma y delta), y otorgando el tiempo necesario para que completasen esta primera fase. Al finalizar y entregar el cuadernillo 1, cada alumno recibía el cuadernillo 2 correspondiente a su modelo, y complementaba así la segunda fase de la aplicación del instrumento de investigación.

Posteriormente, el centro facilitó las calificaciones de los estudiantes correspondientes a la segunda evaluación anual de Matemáticas y Lengua; información fundamental de cara a analizar la relación entre el control de la comprensión y el rendimiento académico, como se propone en los objetivos de la investigación. 
Este estudio piloto de investigación corresponde a un diseño descriptivo, correlacional y transversal.

\section{Resultados}

Previo al análisis definitivo de la información, se calculó el coeficiente Kappa para mostrar el nivel de concordancia entre la medida de CC (tabla VIII) asignada a cada alumno -partiendo de sus respuestas y de las categorías propuestas en la tabla Votorgadas por dos observadores independientes. Los valores de índice Kappa obtenidos para los diferentes cursos y textos evidencian un nivel de concordancia, en todos los casos "sustancial” y “casi perfecto” ( $\mathrm{p}<.01)$ de acuerdo a la nominación de Landis y Koch (1977).

El coeficiente de fiabilidad (alfa de Cronbach) calculado para el instrumento es de 0,790, lo cual indica un nivel de consistencia interna de la prueba alto y con ello, su fiabilidad para a medir el control de la comprensión del alumnado participante en este estudio. La tabla VIII resume los valores obtenidos de dicho parámetro.

\begin{tabular}{cc}
\hline $\begin{array}{c}\text { Puntuación de control de } \\
\text { comprensión por texto }\end{array}$ & $\begin{array}{c}\text { Alfa de Cronbach si se } \\
\text { elimina el elemento }\end{array}$ \\
\hline Texto 1 & 0,749 \\
\hline Texto 3 & 0,742 \\
\hline Texto 4 & 0,644 \\
\hline
\end{tabular}

Tabla VIII. Fiabilidad del instrumento.

\section{En relación al objetivo 1}

\section{Porcentajes de control de la comprensión por cursos}

Para facilitar la comprensión de resultados y lograr que cada sujeto quede adscrito a uno de los tres tipos de CC, de acuerdo con los componentes de evaluación y regulación propuestos por Campanario y Otero (1990), las seis categorías de análisis mencionadas en la tabla $\mathrm{V}$ se han agrupado en tres. Las categorías de análisis definitivas se presentan de la siguiente forma: Conocimiento ilusorio (CI) + Dificultades básicas (DB) conforman la categoría evaluación inadecuada y regulación ausente; Falta de regulación (FR) + Regulación inadecuada (RI), conforman la evaluación adecuada pero regulación de la comprensión inadecuada; y por último, Dificultad infravalorada (DI) + Detección y rechazo (DR) conforman evaluación y regulación de la comprensión adecuadas. Se presentan en la tabla IX los porcentajes de control de la comprensión de los estudiantes, por curso y categorías agrupadas. 


\begin{tabular}{|c|c|c|c|c|}
\hline $\begin{array}{l}\text { Nivel del control } \\
\text { de la } \\
\text { comprensión }\end{array}$ & $N=139$ & $1^{\circ} \mathrm{ESO}$ & $3^{\circ} \mathrm{ESO}$ & $1^{\circ} \mathrm{BACH}$ \\
\hline $\begin{array}{l}- \\
i\end{array}$ & $\begin{array}{l}\text { Evaluación inadecuada y } \\
\text { regulación ausente }\end{array}$ & $35 \%$ & $45,40 \%$ & $26,70 \%$ \\
\hline+ & $\begin{array}{l}\text { Evaluación adecuada pero } \\
\text { regulación inadecuada }\end{array}$ & $35,80 \%$ & $31 \%$ & $39 \%$ \\
\hline & $\begin{array}{c}\text { Evaluación y regulación } \\
\text { adecuada }\end{array}$ & $29,30 \%$ & $23,60 \%$ & $34,30 \%$ \\
\hline
\end{tabular}

Tabla IX. Porcentaje de control de comprensión, según categorías agrupadas y curso.

Como se aprecia, el porcentaje mayor de estudiantes que ni evalúan ni regulan corresponde a $3^{\circ}$ de la ESO, seguido por $1^{\circ}$ ESO y Bachillerato. Llama la atención entre estos cursos, que el 45,4\% de alumnos de $3^{\circ}$ ESO presente una evaluación inadecuada y ausencia de regulación de la comprensión a la hora de valorar los textos propuestos por medio del instrumento. En este sentido, y a diferencia de los resultados de otros autores (Otero y Campanario, 1990; Mazzitelli et al., 2007) en los que el grupo de menor edad presenta los niveles de comprensión más bajos, en este experimento se observa que es el grupo de edad intermedia (dentro del rango considerado en este estudio) el que presenta valores más bajos de comprensión, lo que implica un uso ausente o ineficaz de estrategias de control de la comprensión.

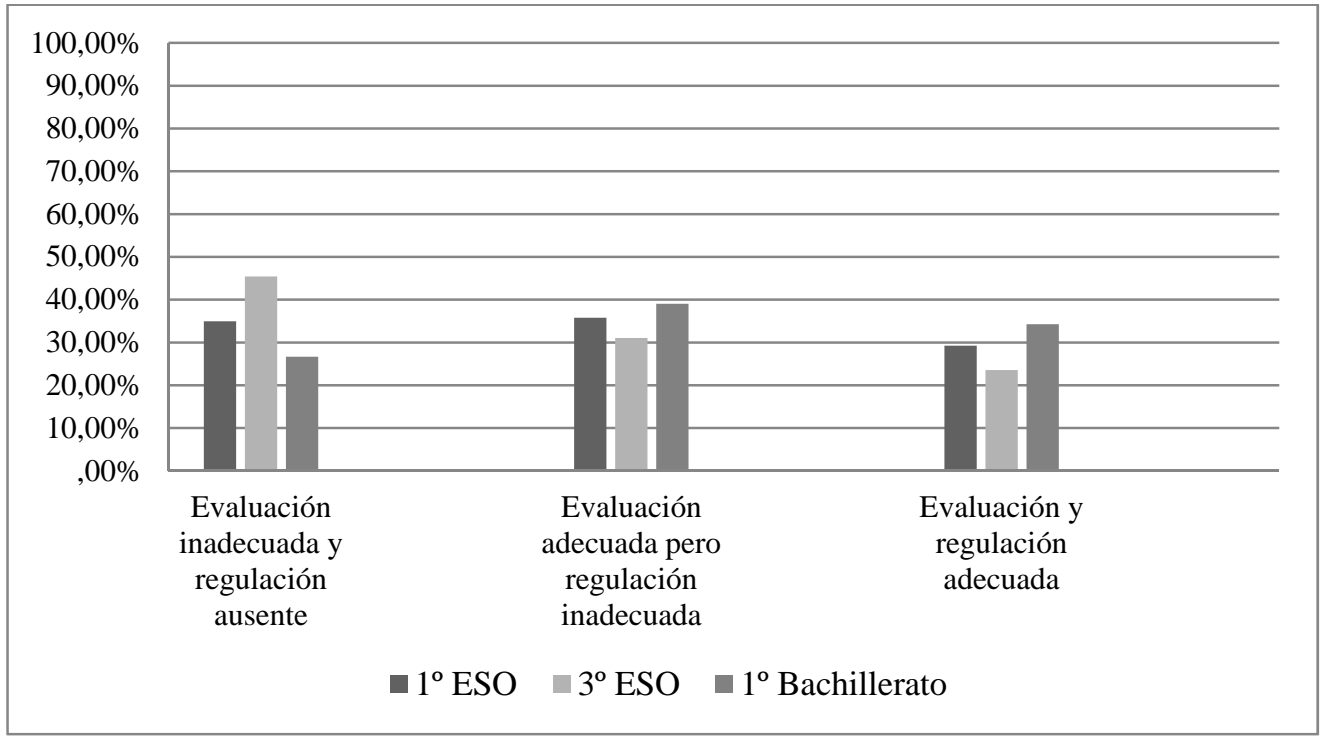

Figura I. Categorías de control de comprensión, por cursos. 
En este primer grupo correspondiente a aquellos que evalúan de forma inadecuada y con regulación ausente, y en el que los sujetos admiten no haber detectado la contradicción, los estudiantes han manifestado diferentes dificultades. Por ejemplo, de tipo conceptual, comentando "no entiendo qué clase de depósito se forma", o de tipo léxicas como "no entiendo filogenia". Además se han dado casos en que manifestaban "no lo puse porque (...) no leí con atención porque estos textos no me llaman la atención", revelando un claro desinterés hacia la tarea y una estrecha relación con el componente motivacional. Finalmente, la mayor proporción de comentarios aludían a cuestiones del tipo "no me di cuenta" o "lo leí muy rápido", poniendo de manifiesto la escasa aplicación de estrategias de monitoreo en la lectura.

Con respecto a los comportamientos que implican una evaluación adecuada pero regulación inadecuada, son los alumnos de mayor edad los que presentan un mayor porcentaje, tal y como evidencia la tabla anterior. En este segundo grupo los estudiantes han señalado dificultades de tipo léxico como "no entiendo que es $A D N$ ribosomal", o de tipo conceptual comentando "es posible que algunas produzcan Oxígeno y otras casi no", o también dificultades que evidenciaban confusión con respecto a las demandas de la tarea (Defior, 2000) como "debido a que creía que había que entender el texto y no corregirlo".

En otras ocasiones, el lector discurre que hay un problema pero no es capaz de discernir su naturaleza diciendo "me da la impresión que se contradice". Finalmente, se ha observado un número considerable de estudiantes que admitían no haber señalado la contradicción, aun aceptando que la habían detectado, estableciendo una relación causal con la autoridad epistémica; es decir, el pudor a dudar de la palabra escrita, explicando por ejemplo "pensé que era una errata del texto".

Por último, el grupo que evidenció una evaluación y regulación adecuadas de acuerdo a las frecuencias aportadas en la tabla IX, corresponde a los alumnos de mayor edad, resultados que están en consonancia con otras investigaciones (Otero y Campanario, 1990; Mazzitelli et al., 2007).

Porcentajes de control de la comprensión por género

Se ha evidenciado una evaluación y regulación de la comprensión ausente e inadecuada, principalmente en las mujeres; mientras que en los hombres el mayor porcentaje corresponde a una evaluación adecuada aunque una regulación ausente o inadecuada. La tabla X y figura II evidencian los diferentes porcentajes de las categorías agrupadas por género. 


\begin{tabular}{|c|c|c|c|}
\hline $\begin{array}{l}\text { Nivel del control } \\
\text { de la } \\
\text { comprensión }\end{array}$ & $\mathrm{N}=139$ & Hombres & Mujeres \\
\hline \multirow[t]{2}{*}{ - } & $\begin{array}{l}\text { Evaluación inadecuada y } \\
\text { regulación ausente }\end{array}$ & $29,50 \%$ & $45,60 \%$ \\
\hline & $\begin{array}{l}\text { Evaluación adecuada pero } \\
\text { regulación inadecuada }\end{array}$ & $41,10 \%$ & $27,70 \%$ \\
\hline+ & $\begin{array}{c}\text { Evaluación y regulación } \\
\text { adecuada }\end{array}$ & $29,50 \%$ & $26,70 \%$ \\
\hline
\end{tabular}

Tabla X. Distribución de frecuencias de alumnos, según categoría y género.

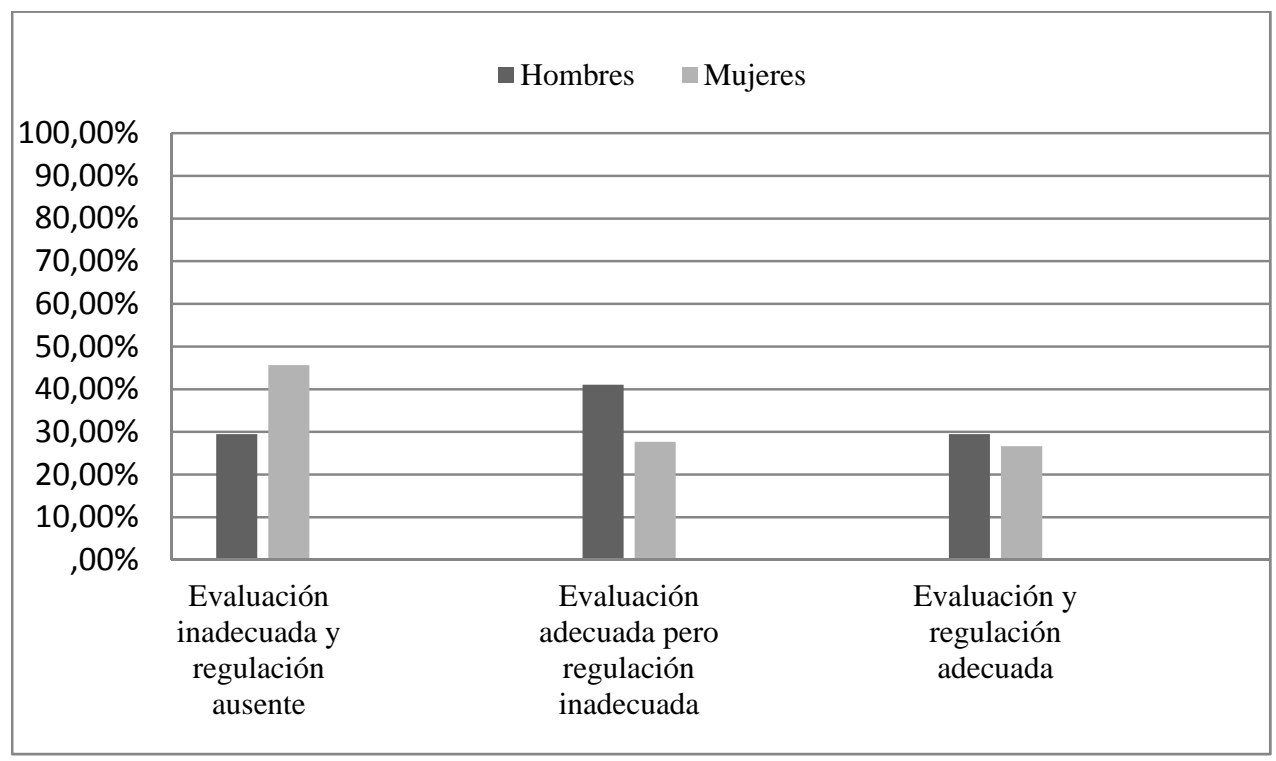

Figura II. Niveles de CC según género.

En el caso de los hombres, tal y como se ha citado, el porcentaje mayor se detecta en la categoría evaluación adecuada pero regulación inadecuada. Cuando se le pide al lector que explique las razones de no haber señalado ni subrayado las contradicciones, aun cuando reconoce haber detectado la inconsistencia, el grupo masculino hace referencia a dificultades diversas, siendo muy frecuentes aquellas relacionadas con confusiones respecto a las demandas de la tarea con explicaciones del tipo "Porque no preguntaba ni decía nada de señalarlo, sólo me he limitado a responder". También 
han sido reiteradas las explicaciones en las que, tal como afirma Otero y Campanario (1990), el lector resta importancia al problema con argumentos de tipo "me di cuenta pero no pensé que era necesario indicarlo", manifestando un comportamiento en el que el sujeto identifica una complicación (evalúa) pero luego no pone en marcha mecanismos de reparación (no regula) y resuelve la situación minimizando los problemas encontrados.

Además, se han encontrado numerosos casos es los que el lector falla en su monitorización, ya que, aunque se da cuenta de la contradicción, no rechaza el texto, que sería lo deseable, a causa del respeto a la palabra escrita (autoridad epistémica), las explicaciones han sido del tipo "porque siendo que estaba aquí escrito, me extrañaba, pero pensé que seguramente esté bien, aunque yo no lo creía".

Se han encontrado otros casos en los que el lector si regula, pero de forma errónea, explicando así la inconsistencia en uno de ellos, (es) "porque creí que el agua era un líquido especial". Finalmente, algunos han interpretado que las inconsistencias habían sido propuestas deliberadamente y que la función de la prueba era tratar de engañar o probar al sujeto; esto corresponde a una interpretación pragmática de la función de ese texto, por ejemplo la explicación "pienso que lo han puesto dos veces aposta".

En las mujeres, el mayor porcentaje se halla en la categoría evaluación inadecuada y ausencia de regulación, mientras que el menor porcentaje se presenta en la categoría de evaluación y regulación adecuada.

Las dificultades halladas por el grupo de mujeres que ni evalúan ni regulan han sido de tipo léxico y conceptual, con argumentaciones como "utiliza un lenguaje científico y hay cosas que no se entiende" o "no sé que es filogenia".

Aquellas alumnas que sí afirman haber detectado la contradicción pero ni señalan ni explican el problema han indicado, hasta en diez ocasiones, que era una errata del texto haciendo alusión a la autoridad epistémica que les impide hacer juicos de valor sobre la palabra escrita. También se han observado argumentos que restan importancia al hecho de no señalar la contradicción con explicaciones del tipo "No le di verdadera importancia, para señalarlo".

En varias ocasiones las alumnas han detectado algo extraño en el texto pero no son capaces de discernir su naturaleza manifestando explicaciones del tipo "me confundí un росо"; asimismo, han evidenciado confusiones en relación a las demandas de la tarea, con explicaciones del tipo "no pensé que fueran contradicciones".

También se han encontrado casos en los que sí se evalúa y regula, pero con inferencias incorrectas, es ejemplo la explicación dada por una alumna que argumenta "porque pensé que, al ser submarinas, el oxígeno no servía de mucho".

\section{En relación al objetivo 2}

Para contrastar la independencia entre el CC y las variables curso y género es necesario partir de una muestra cuyas observaciones sean independientes, por lo cual se ha analizado cada texto por separado manejando de forma agrupada las tres 
categorías a antes explicadas. Se han realizado pruebas Chi-cuadrado de Pearson para cada variable.

\section{Relación CC y curso}

A pesar de que se han evidenciado diferencias por curso en el CC en los porcentajes por categorías antes comentados, la realización de la prueba Chi-cuadrado muestra que no hay diferencias estadísticamente significativas entre CC y curso, como se aprecia en la Tabla XI.

\begin{tabular}{cc}
\hline $\begin{array}{c}\text { Nivel de comprensión por } \\
\text { texto }\end{array}$ & Significatividad \\
\hline Texto 1 & 0,183 \\
\hline Texto 3 & 0,604 \\
\hline Texto 4 & 0,373 \\
\hline
\end{tabular}

Tabla XI. Pruebas Chi-Cuadrado de Pearson por curso.

Se han realizado además análisis estadísticos intracursos para los grupos de $1^{\circ}$ de ESO A y B, y para los grupos de $3^{\circ}$ de ESO A y B. Los resultados se resumen en la Tabla XII. En el caso de $1^{\circ}$ de ESO no se han encontrado pruebas que evidencien que haya diferencias en los grupos en cuanto a los niveles de CC. Asimismo, la cercanía de los p-valores a valores significativos (p-valor $<.05)$ podrían indicar que si la muestra fuera algo mayor se lograría probar tales diferencias.

\begin{tabular}{ccc}
\hline Nivel de comprensión por texto & $\mathbf{1}^{\mathbf{0}}$ ESO Sig. & $\mathbf{3}^{\mathbf{0}}$ ESO Sig. \\
\hline Texto $\mathbf{1}$ & 0,12 & 0,69 \\
\hline Texto $\mathbf{3}$ & 0,161 & 0,825 \\
\hline Texto 4 & 0,072 & 0,479 \\
\hline
\end{tabular}

Tabla XII. Prueba Chi-cuadrado intracursos.

Relación CC y género

A pesar de que se han evidenciado diferencias por género en el CC en los porcentajes previamente mencionados, la realización de la prueba Chi-cuadrado ha demostrado que no hay diferencias estadísticamente significativas entre CC y género, como se aprecia en la Tabla XIII. En el texto 3, en particular, no se han encontrado diferencias significativas según el género, aunque la significatividad se acerca al pvalor de 0.1 . 
Nivel de comprensión por texto Significatividad

\begin{tabular}{cc}
\hline Texto 1 & 0,788 \\
\hline Texto 3 & 0,102 \\
\hline Texto 4 & 0,204 \\
\hline
\end{tabular}

Tabla XIII. Pruebas Chi-cuadrado por género.

\section{En relación al objetivo 3}

\section{Relación entre comprensión de textos y rendimiento escolar}

Este apartado se centra averiguar si las correlaciones entre CC y las calificaciones en asignaturas como Lengua, Matemáticas, y ambas, pudieran estar asociadas significativamente, y por ende establecer una relación con el rendimiento escolar. Para afinar el cálculo estadístico se han tipificado tanto el CC (la media de los CC de los tres textos) como las calificaciones dentro de cada grupo.

\begin{tabular}{cccc}
\hline & $\mathbf{1}^{\mathbf{0}}$ ESO & $\mathbf{3}^{\mathbf{0}}$ ESO & $\mathbf{1}^{\mathbf{0}} \mathbf{B A C H}$ \\
\hline CC-Mat & $0,381^{* *}$ & 0,114 & $-0,026$ \\
\hline CC- Lengua & $0,433^{* *}$ & 0,148 & 0,109 \\
\hline CC- Media & $0,443^{* *}$ & 0,145 & 0,049 \\
\hline \multirow{4}{*}{$* *$ La correlación es significativa al nivel .01 }
\end{tabular}

Tabla XIV. Coeficientes de correlación de Pearson entre CC tipificado por medias y curso.

Como refleja la tabla XIV, tan solo en $1^{\circ}$ de ESO las correlaciones son positivas y significativas con un valor moderado. En el resto, los valores demuestran una asociación muy débil y no significativa. El hecho de no encontrar una asociación fuerte en ningún curso, junto con la disminución progresiva de los valores según avanza la edad de los alumnos, refuerza la influencia limitada que tiene el CC en el rendimiento académico. Esta limitación ya había sido apuntada en trabajos previos (García-Arista et al., 2006).

Cuando se reagrupa la muestra en función del género y no por curso, tan solo se encuentran diferencias significativas en el grupo de los varones, y en concreto entre el CC y las calificaciones de Lengua y calificaciones medias (Lengua + Matemáticas), mientras que en el grupo de las mujeres la asociación es muy débil y no significativa. 


\begin{tabular}{ccc}
\hline & Varones & Mujeres \\
\hline CC- Mat & 0,145 & 0,173 \\
\hline CC- Lengua & $0,285^{*}$ & 0,208 \\
\hline CC- Media calificaciones & $0,239^{*}$ & 0,212 \\
\hline
\end{tabular}

* La correlación es significante al nivel .05

Tabla XV. Coeficientes de correlación (Pearson) entre el control de la comprensión (CC) (tipificado) y el rendimiento académico en las asignaturas de Matemáticas (Mat), Lengua y media de ambas, según el género.

\section{Discusión}

Escudero y León (2007) afirman que una de las formas en las que se utiliza el texto en el proceso de Enseñanza-Aprendizaje de las Ciencias tiene como finalidad que el nivel de conocimientos del lector aumente con la lectura del mismo. Por tanto, es necesario que el educando comprenda lo que está leyendo y si no es así, que sea capaz de darse cuenta de que no entiende y ponga en marcha estrategias para solucionar el problema de comprensión. Aprender requiere de los sujetos, la aplicación de estrategias en las que el control y regulación de los procesos cognitivos son imprescindibles, ya que juegan un rol determinante en la competencia de "aprender a aprender”, eje central de la educación actual. Asimismo, estas estrategias influyen en el aprendizaje autorregulado, puesto que junto con otras variables (motivacionales y conductuales) definen a un alumno activo con su formación y orientado hacia la consecución de metas y el aprendizaje permanente, atributos imprescindibles en el marco europeo de educación.

En este sentido, y a pesar de la manifiesta trascendencia que tiene la adquisición de las competencias básicas en general, la realidad es que las evaluaciones llevadas a cabo por organismos internacionales ponen de manifiesto la necesidad acuciante de mejorar los niveles de ciencias y lengua en nuestras aulas. Según datos arrojados en diversas ediciones del Informe PISA (2003, 2006, 2009), los estudiantes presentan dificultades para comprender, utilizar y reflexionar sobre textos escritos, a la par que relacionar y aplicar el conocimiento científico (OCDE 2005; 2006; 2010).

En la presente investigación se ha evaluado la capacidad metacognitiva de estudiantes de ESO y Bachillerato para detectar la existencia de contradicciones (criterio de consistencia interna) y establecer mecanismos de regulación en la lectura de textos científicos manipulados. Los resultados apuntan a un déficit de habilidades metacognitivas en relación a la lectura Tal y como se ha mencionado la falta de autorregulación afecta la comprensión lectora y esta puede influir en el desarrollo de otras capacidades como puede ser el aprendizaje autónomo y la transferencia de conocimiento (Guevara y Guerra, 2013). Se han observado diferencias, aunque no 
significativas, en el CC según la edad de los estudiantes. Aunque se esperaba que fueran los alumnos de menor edad los que presentaran valores más bajos de CC, esto no ha sido así. El mayor porcentaje de estudiantes con valores de CC menor, es decir, que ni siquiera han detectado las contradicciones, ha recaído en los estudiantes pertenecientes al curso de $3^{\circ}$ de ESO.

También se han encontrado diferencias considerables (más de 15 puntos), pero no significativas, entre el CC en chicos y chicas. Son ellas las que han mostrado una monitorización más defectuosa, pues casi la mitad de la muestra no ha detectado las contradicciones, frente a un tercio de los chicos. Estos porcentajes se invierten en el siguiente grado de CC, es decir, casi la mitad de la muestra de chicos han identificado las contradicciones aunque no las han señalado ni las han explicado, frente a un tercio de chicas. Por el contrario, los porcentajes están prácticamente a la par cuando la monitorización ha sido la deseada, es decir, cuando se han detectado las contradicciones y se han señalado explícitamente como un problema. Estos hallazgos reiteran lo indicado anteriormente, ya que únicamente un tercio de la muestra presenta un control de la comprensión lectora adecuado.

En relación al componente regulador del CC, las principales explicaciones manifestadas por los alumnos hacen alusión a dificultades respecto a confusiones con las demandas de la tarea, el respeto a la autoridad epistémica y la minimización del problema. En el primer caso, se refiere a la falta de conciencia de cuál es la finalidad de la lectura; así los lectores poco competentes creen que para comprender el significado del texto basta con decodificar las palabras limitando el proceso de lectura a únicamente el nivel léxico. Son las chicas las que han argumentado en mayor proporción este tipo de dificultad. En el segundo caso, referente al respeto a la autoridad epistémica, son diversas las investigaciones que han obtenidos resultados similares (Otero y Campanario, 1990; Mazzitelli et al., 2007); lo que implica la necesidad en este sentido de formar estudiantes capaces de discrepar de la palabra escrita y desarrollar el juicio crítico, aspecto que para los lectores se establece como una finalidad importante de la metacognición. Esta argumentación ha sido frecuente en toda la muestra, independientemente del género. Finalmente, los chicos, en su mayoría, han minimizado la importancia de no señalar ni explicar las contradicciones, lo que revela una monitorización defectuosa; el alumno se da cuenta de la dificultad pero no cree que sea importante reparar tal dificultad y, por tanto, no lleva a cabo ninguna estrategia para mejorar la comprensión. Esto resulta trascendental, pues se podrá alcanzar una representación coherente de lo que se lee una vez que las inconsistencias sean no solo detectadas, sino también corregidas durante el proceso de control.

De otra parte, Guevara y Guerra (2013) afirman que una deficiente comprensión lectora repercute en el rendimiento académico. Entre los factores que influyen en las dificultades de comprensión lectora se halla el escaso control y dirección del proceso lector (estrategias metacognitivas). Sin embargo, investigaciones previas (Otero et al., 1992; García-Arista et al., 2006) han hallado correlaciones débiles entre CC y rendimiento académico. Los resultados encontrados en esta investigación corroboran estos estudios, pues muestran correlaciones muy débiles que decrecen con la edad. Es decir, es entre los estudiantes de $1^{\circ}$ de ESO donde un mayor CC va asociado a un 
mejor rendimiento académico (medido según las calificaciones escolares), mientras que en $1^{\circ}$ de Bachillerato no se ha encontrado esta relación. Se apoya, por tanto, la interpretación dada por García-Arista et al. (2006) que atribuye esta escasa relación entre CC y rendimiento académico al modelo de evaluación que se utiliza en nuestro sistema educativo, donde se premia el aprendizaje memorístico (Pozo y Gómez, 2009) en detrimento de un aprendizaje significativo. Por tanto, el papel que deben tener el uso de estrategias metacognitivas en los estudiantes puede quedar comprometido y eludido por un modelo de evaluación que no requiere de estas habilidades para alcanzar las metas propuestas. Es decir, si el alumno goza de éxito académico sin tener que desarrollar y emprender estrategias de control de la comprensión, no invertirá esfuerzo ni tiempo en adquirir dichas destrezas. Resulta, por ello, imprescindible introducir métodos de evaluación en un contexto de educación personalizada que valoren un aprendizaje significativo acorde con las demandas de la sociedad actual. Finalmente, es oportuno destacar la similitud de los resultados hallados en esta investigación con la llevada a cabo por Otero et al. (1992). Esto evidencia la escasa mejoría conseguida y la necesidad de emprender acciones en el aula encaminadas a mejorar las estrategias metacognitivas de los educandos.

\section{Conclusiones}

La muestra evaluada ha mostrado escaso control de la comprensión siendo este más bajo en las chicas frente a los chicos, y en el curso de $3^{\circ}$ de la ESO, frente a $1^{\circ}$ de la ESO y $1^{\circ}$ de Bachillerato.

Los estudios estadísticos realizados no han evidenciado diferencias significativas entre las variables de género o curso, aunque la proximidad a valores significativos apunta a que con muestras de mayor tamaño los resultados sí podrían ser significativos.

Las correlaciones realizadas entre rendimiento académico y CC han arrojado una asociación débil e inversamente proporcional a la edad de los educandos, resultados que concuerdan con los estudios previos.

\section{Agradecimientos}

A Blanca Arteaga, Investigadora principal del GdI Educación Personalizada en la Era Digital (EPEDIG) de la Universidad Internacional de La Rioja y a José Otero profesor Emérito de la Universidad de Alcalá.

\section{Referencias bibliográficas}

ALEXANDER, P. A. Y KUIKOWICH J. M. (1994). Learning form Physics text: a syntehsis of recent research. Journal of Research in Science Teaching, 31 (9), 895911.

BAKER, L. (1985). How do we know when we don't understand? Standards for evaluating text comprehension. En D.L. Forrest-Pressley, G.E. Mackinnon, T.G. 
Waller, (Eds.). Metacognition, cognition and human performance. Nueva York: Academic Press.

BROC, M. A. (2012) Influencia relativa de variables metacognitivas y volitivas en el rendimiento académico de estudiantes de Bachillerato (LOE). REOP, 23 (3), 63-80. Recuperado de: http:/espacio.uned.es/revistasuned/index.php/reop/article/view/11462/10959

CAMPANARIO, J. M. y MOYA, A. (1999). ¿Cómo enseñar ciencias? Principales tendencias y propuestas. Enseñanza de las ciencias, 17 (2), 179-192.

CANO, F. (2005). Epistemiological beliefs and approaches to learning: Their change through secondary school and their influence on academic performance. British Journal of Educational Psycology, 75, 203-221.

COOPER, J. D. (1990). Cómo mejorar la comprensión lectora. Madrid: AprendizajeVisor.

DEFIOR, S. (2000). Las dificultades de aprendizaje: un enfoque cognitivo. Lectura, escritura, matemática. Málaga: Aljibe.

ESCUDERO, I. y LEÓN, J. A. (2007). Procesos inferenciales en la comprensión del discurso escrito: Influencia de la estructura del texto en los procesos de comprensión. Revista Signos, 40 (64), 311-336. Recuperado de http://www.scielo.cl/scielo.php?script=sci_arttext\&pid=S0718-

$09342007000200003 \& \operatorname{lng}=$ es\&tlng=es. $10.4067 /$ S0718-09342007000200003

FLAVELL, J. H. (1976). Metacognitive aspects of problem solving. En L. B. Resnik (Eds.). The nature of intelligence (pp. 231-235). Hillsdale, N. J.: Erlbaum.

GARCÍA-ARISTA, E.; OTERO, J.; CALDEIRA, M.H.; PRATA-PINA, E.; PATRICIO, A. y CARDOSO, E. (2006). El control de la comprensión y el rendimiento académico en ciencias de los alumnos españoles y portugueses. En J. Díaz de Bustamante, y M. P. Jiménez Aleixandre, (Eds.). Perspectivas sobre a aprendizaxe das Ciencias e das Matemáticas. Estudios en honor al profesor Eugenio García-Rodeja Fernández (pp. 83-95). Santiago de Compostela: Servicio de Publicaciones de la Universidad de Santiago.

GEORGHIADES, P. (2004). From the general to the situated: three decades of metacognition. International Journal of Science Education, 26, 365-383.

GUEVARA, Y. y GUERRA J. (2013). La comprensión lectora como competencia genérica. Revista electrónica de Psicología Iztacala, 16 (2), 320-339. Recuperado de: http://www.medigraphic.com/pdfs/epsicologia/epi-2013/epi132b.pdf

LANDIS, J. R. y KOCH, G. G. (1977). The measurement of observer agreement for categorical data. Biometrics, 33, 159-174.

LÓPEZ, S. A. D. (2007). Establecimiento de una metaestrategia de aprendizaje en la formación del psicólogo. Enseñanza e investigación en psicología, 12, 15-25. 
MAZZITELLI, C. A., MATURANO, C. I. y Macías, A. (2007). Estrategias de monitoreo de la comprensión en la lectura de textos de ciencias con dificultades. Enseñanza de las Ciencias, 25 (2), 217-228.

MOKHTARI, K. y REICHARD C. A. (2002). Assessing students'metacognitive awareness of Reading strategies. Journal odf Educational Psycology, 94 (2), 249259.

MUÑOZ-CALVO. E., MUÑOZ-MUÑOZ, L., GARCIA, M. C. Y GRANADO, L. A (2013). La comprensión lectora de textos científicos en el proceso de enseñanzaaprendizaje. Humanidades Médicas, 13(3), 772-804.

OCDE (2005). Informe PISA 2003. Aprender para el mundo de mañana. Madrid: Santillana. Recuperado de: http://www.oecd.org/pisa/39732493.pdf

OCDE (2006). PISA 2006. Marco de la Evaluación. Conocimientos y habilidades en Ciencias, Matemáticas y Lectura. Madrid: Santillana Recuperado de:

http://www.oecd.org/pisa/39732471.pdf

OCDE (2010). PISA 2009 Results: What makes a School Successful? Resources, Policies and

practices. Paris: OCDE. Recuperado de: http://www.oecd.org/pisa/pisaproducts/48852721.pdf

OTERO, J. (1992). El aprendizaje receptivo de las ciencias: preconcepciones, estrategias cognitivas y estrategias metacognitivas. Tarbiya, 1 (2), 57-65.

OTERO, J. C. (1998) Influence of knowledge activiation and context on comprenhension monitoring id science texts. En D. Hacker, J. Dunlosky y A. Graesser (Eds.). Metacognition in educational theory and practice (pp. 145-164). Mahwah, New Jersey: Eribaum Associates publishers.

OTERO, J. y CAMPANARIO, J. M. (1990). Comprehension evaluation and regulation in learning from science texts. Journal of research in science teaching, 27 (5), 447460 .

OTERO, J., CAMPANARIO, J. M. y HOPKINS, K. (1992). The relatioship between academic achievement and metacognitive comprenhension monitoring ability of spanish Secondary School Students. Educational and psychological measurement, 52, 419-430.

OTERO J. C. y GRAESSER A.C. (2001). PREG: Elements of a model of question. Cognition and instruction, 19, 143-175.

PINTRICH P. y DE GROOT E. V. (1990). Motivational and self-regulated learning components of classroom academic performance. Journal of Educational Phycology, 82, 33-40.

POZO, J. I. y Gómez, M. A. (2009). Aprender y enseñar ciencia. Del conocimiento cotidiano al conocimiento científico (6a ed.). Madrid: Morata. 
RODRÍGUEZ, G. (2009). Motivación, estrategias de aprendizaje y rendimiento académico en estudiantes de ESO. (Tesis Doctoral). Universidad de A Coruña, A Coruña. de: http://ruc.udc.es/dspace/bitstream/2183/5669/1/RodriguezFuentes_Gustavo.TESIS_ GRF_210109.pdf

SANJOSÉ, V., FERNÁNDEZ, J.J. Y VIDAL-ABARCA, E. (2010). Importancia de las destrezas de procesamiento de la información en la comprensión de textos científicos. Infancia y Aprendizaje, 33(4), 529-541.

SCHUNK, D. H. y ZIMMERMAN, B. (1994). Self-regulation in education: Retrospect and prospect. En D. H. Schunk y B. Zimmerman, (Eds.), Self-regulation of learning and performance: Issues and educational (pp. 305-314). Hillsdale: Lawrence Erlbaum Associates.

SOLAZ, J. y SANJOSÉ, V. (2008). Conocimientos y procesos cognitivos en la resolución de problemas de ciencias: consecuencias para la enseñanza. Magis, Revista Internacional de Investigación en Educación, 1, 147-162. Recuperado de: http://revistas.javeriana.edu.co/index.php/MAGIS/article/view/3361/2566

WASHER, P. (2007). Revisiting key skills: a practical framework for higher education. Quality in Higher education, 13 (1), 57-67.

WINOGRAD, P. y JOHNSTON, P. (1982) Comprehension monitoring and the error detection paradigm. Journal of Reading Behavior, 14, 61-76.

ZIMMERMAN, B. J., y MARTÍNEZ-PONS, M. (1988). Construct validation of a strategy model of student self-regulated learning. Journal of Educational Psychology, 80, 284-290.

\section{Correspondencia con los autores}

Lourdes JIMÉNEZ TARACIDO

Universidad Internacional de la Rioja

Gran Vía Rey Juan Carlos I, 41, 26002

Logroño (La Rioja), España.

e-mail: lourdes.jimenez@unir.net

Daniela BARIDON CHAUVIE

Universidad Internacional de la Rioja

Gran Vía Rey Juan Carlos I, 41, 26002

Logroño (La Rioja), España.

e-mail: daniela.baridon@unir.net

Ana Isabel MANZANAL MARTÍNEZ

Universidad Internacional de la Rioja

Gran Vía Rey Juan Carlos I, 41, 26002

Logroño (La Rioja), España.

e-mail: ana.manzanal@unir.net 